University of South Florida

DIGITAL COMMONS

Digital Commons @ University of

@ UNIVERSITY OF SOUTH FLORIDA

South Florida

USF Tampa Graduate Theses and Dissertations

USF Graduate Theses and Dissertations

2006

\title{
Crossing locations, light conditions, and pedestrian injury severity
}

Naved Alam Siddiqui

University of South Florida

Follow this and additional works at: https://digitalcommons.usf.edu/etd

Part of the American Studies Commons

\section{Scholar Commons Citation}

Siddiqui, Naved Alam, "Crossing locations, light conditions, and pedestrian injury severity" (2006). USF Tampa Graduate Theses and Dissertations.

https://digitalcommons.usf.edu/etd/2701

This Thesis is brought to you for free and open access by the USF Graduate Theses and Dissertations at Digital Commons @ University of South Florida. It has been accepted for inclusion in USF Tampa Graduate Theses and Dissertations by an authorized administrator of Digital Commons @ University of South Florida. For more information, please contact digitalcommons@usf.edu. 
Crossing Locations, Light Conditions, and

Pedestrian Injury Severity

by

Naved Alam Siddiqui

A thesis submitted in partial fulfillment of the requirements for the degree of

Master of Science in Civil Engineering

Department of Civil and Environmental Engineering

College of Engineering University of South Florida

Co-Major Professor: Ram Pendyala, Ph.D.

Co-Major Professor: Xuehao Chu, Ph.D.

Elaine Chang, Ph.D.

Jian John Lu, Ph.D.

Date of Approval:

May 30, 2006

Keywords: pedestrian safety, KABCO, midblock locations, ordered probit model, marginal effects

(C) Copyright 2006, Naved Alam Siddiqui 


\section{ACKNOWLEDGEMENT}

I feel extremely happy to express my deep sense of gratitude and sincere thanks to my advisor Dr. Ram Pendyala for his guidance throughout the course of my graduate studies. It is my privilege to extend thanks to Dr. Xuehao Chu who, acted as my guide during my stay here at the Center for Urban Transportation Research (CUTR) and gave me an opportunity to work on some wonderful research projects; one of which is presented here as my thesis. His keen interest

in my research work, generous supervision, and free hand at work could only foster the development of this study.

I would also like to thank Dr. Steve Polzin for providing valuable insights into the study. I am also grateful to the CUTR family specially my office mates for providing me an excellent work environment. Constant support from my friends and room mates is also worth a mention here.

I would also like to take this opportunity to thank the Florida Department of Transportation (FDOT) for providing funding for this study and the Florida Department of Highway Safety and Motor Vehicles (FDHSMV) for providing the crash database used for the study.

At last but by no means least, I would like to express my gratitude towards my parents and family back in India for their constant moral support throughout my stay here in the United States. 
TABLE OF CONTENTS

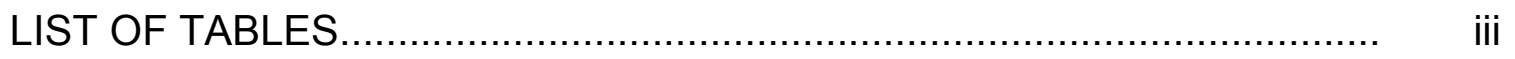

LIST OF FIGURES .......................................................................... iv

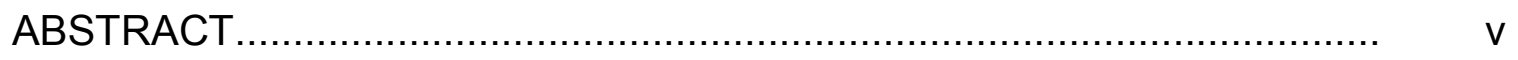

CHAPTER 1: INTRODUCTION .............................................................

CHAPTER 2: CROSSING LOCATIONS, LIGHT CONDITIONS, AND

PEDESTRIAN INJURY SEVERITY................................... 3

2.1. Introduction ....................................................................

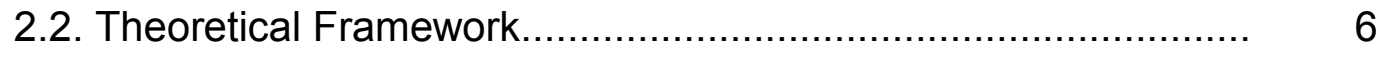

2.2.1. Direct Determinants................................................

2.2.2. Indirect Determinants........................................... 8

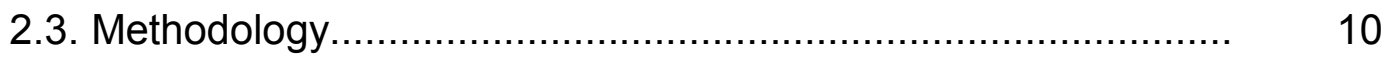

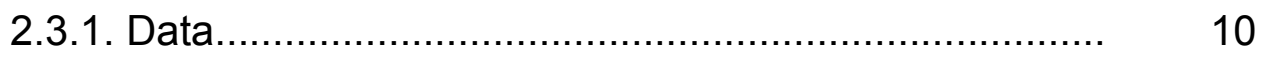

2.3.2. Econometric Model................................................. 12

2.3.3. Model Specification................................................... 14

2.3.3.1. Guidance ............................................. 15

2.3.3.2. Locations and Light Conditions.................... 16

2.3.3.3. Control Variables......................................... 19

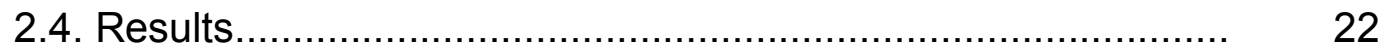

2.4.1. Estimated Model.................................................... 22 
2.4.3. Locations and Light Conditions................................ 26

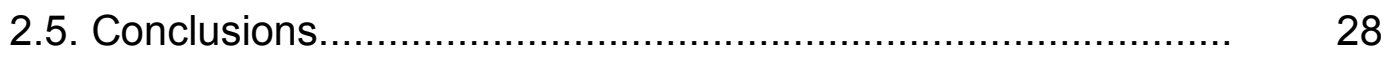

CHAPTER 3: CONCLUSIONS............................................................ 32

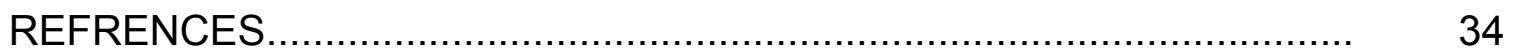

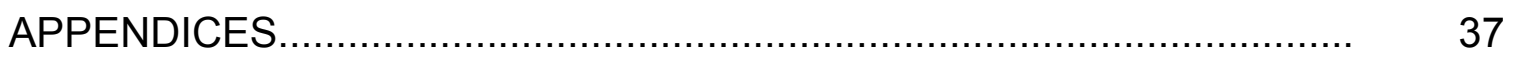

Appendix A: Letter of Acceptance for Publication....................... 38 


\section{LIST OF TABLES}

Table 1: Descriptive Statistics and Expected Direction of Effects...............

Table 2: Ordered Probit Model of Pedestrian Injury Severity..................... 23

Table 3: Marginal Effects on the Probability of a Fatal Injury..................... 24

Table 4: Location Effects: Differences in Probability and Odds of Dying from Crash Involvement between Intersections and Midblock Locations

Table 5: Effects of Daylight and Street Lighting: Differences in Probability and Odds of Dying from Crash Involvement....................................... 


\section{LIST OF FIGURES}

Figure 1: Pedestrian Fatality Risk by Crossing Location and Light Condition

Figure 2: A Framework on the Determinants of Pedestrian Injury Severity.. 6 


\title{
CROSSING LOCATION, LIGHT CONDITIONS, AND PEDESTRIAN INJURY SEVERITY
}

\author{
Naved Alam Siddiqui
}

\begin{abstract}
This study assesses the role of crossing locations and light conditions in pedestrian injury severity through a multivariate regression analysis to control for many other factors that also may influence pedestrian injury severity. Crossing locations include midblock and intersections, and light conditions include daylight, dark with street lighting, and dark without street lighting. The study formulates a theoretical framework on the determinants of pedestrian injury severity, and specifies an empirical model accordingly. An ordered probit model is then applied to the KABCO severity scale of pedestrian injuries which occurred while attempting street crossing in the years 1986 to 2003 in Florida. In terms of crossing locations, the probability of a pedestrian dying when struck by a vehicle, is higher at midblock locations than at intersections for any light condition. In fact, the odds of sustaining a fatal injury is 49 percent lower at intersections than at midblock locations under daylight conditions, 24 percent lower under dark with street lighting conditions, and 5 percent lower under dark without street lighting
\end{abstract}


conditions. Relative to dark conditions without street lighting, daylight reduces the odds of a fatal injury by 75 percent at midblock locations and by 83 percent at intersections, while street lighting reduces the odds by 42 percent at midblock locations and by 54 percent at intersections. 


\section{CHAPTER 1 \\ INTRODUCTION}

This thesis presents a paper titled "Crossing Locations, Light Conditions, and Pedestrian Injury Severity" which has been accepted for publication in the Journal of Transportation Research Record published by Transportation Research Board, Washington D.C. The paper assesses the role of crossing locations on the pedestrian injury severity under different light conditions. The adopted methodology and model specification also gives an opportunity take a quantitative look into the role played by daylight and streetlights in decreasing the severity of the pedestrian crashes.

A quick look at pedestrian crash facts shows that there are far more pedestrian crashes at midblock locations than at the intersection locations. This is expected as the intersection locations consists of just the two end locations while midblock locations are defined as the entire remaining length of the block. However, no previous study has looked into the severity aspect of the pedestrian crashes with respect to the crossing locations. This study performs a comparative study of the injury severity of the pedestrian crashes at midblock versus the intersection locations.

It has been found that, if a pedestrian is struck by a vehicle while crossing the street at a midblock location, he is more likely to sustain a more severe injury 
than when struck while attempting street crossing at intersection locations. This fact remains true under all light conditions: daylight, dark with street lights, as well as dark without street lights. Also, the study quantitatively establishes the role played by light conditions in the injury severity of pedestrians involved in crashes while street crossing.

The study is formulated around a theoretical framework presented in the paper which shows the different factors and their effects on the injury severity of the pedestrian once already stuck by a vehicle. The ordered probit model developed in the study also takes into account a large number of control variables which increases the accuracy of the results obtained for the variables of interest: crossing locations and light conditions. A large database of all pedestrian crashes spanning 18 years from 1986 through 2003 has been used for the analysis.

Chapter 2 of the thesis presents the paper in its entirety followed by the conclusions in Chapter 3. 


\section{CHAPTER 2 \\ CROSSING LOCATIONS, LIGHT CONDITIONS, AND PEDESTRIAN INJURY SEVERITY}

\subsection{Introduction}

In 2003, 4,749 pedestrians were killed and 70,000 injured by motor vehicles in the United States (Traffic Safety Facts, NHTSA, 2003). The average pedestrian fatality rate in the United States in 2003 was 1.63 deaths per 100,000 population (Traffic Safety Facts, NHTSA, 2003). The situation is even more severe in Florida, which had the second highest rate in the country at 2.94 fatalities per 100,000 population.

One serious problem with pedestrian safety in Florida relates to crossing locations. Based on data from 1986 through 2003, nearly 70 percent of pedestrian crashes occurred while the pedestrians were crossing at midblock locations versus intersections. This high percentage may be consistent with the fact that midblock locations span a greater proportion of roadway segments, while intersections consist of only two end-point locations. However, the problem at midblock locations is more than its magnitude. The risk is higher as well. The number of pedestrian fatalities as a ratio to the total number of pedestrians involved in crashes while attempting street crossing is higher at midblock locations than at intersection locations under any light condition (Figure 1). No 
existing research is found that compares pedestrian injury severity risks associated with midblock and intersection locations. In a recent study, Lee and Abdel-Aty analyzed pedestrian crashes at intersection locations in Florida for 1999-2002, but they did not perform any comparative study between locations (Lee and Abdel-Aty, 2005).

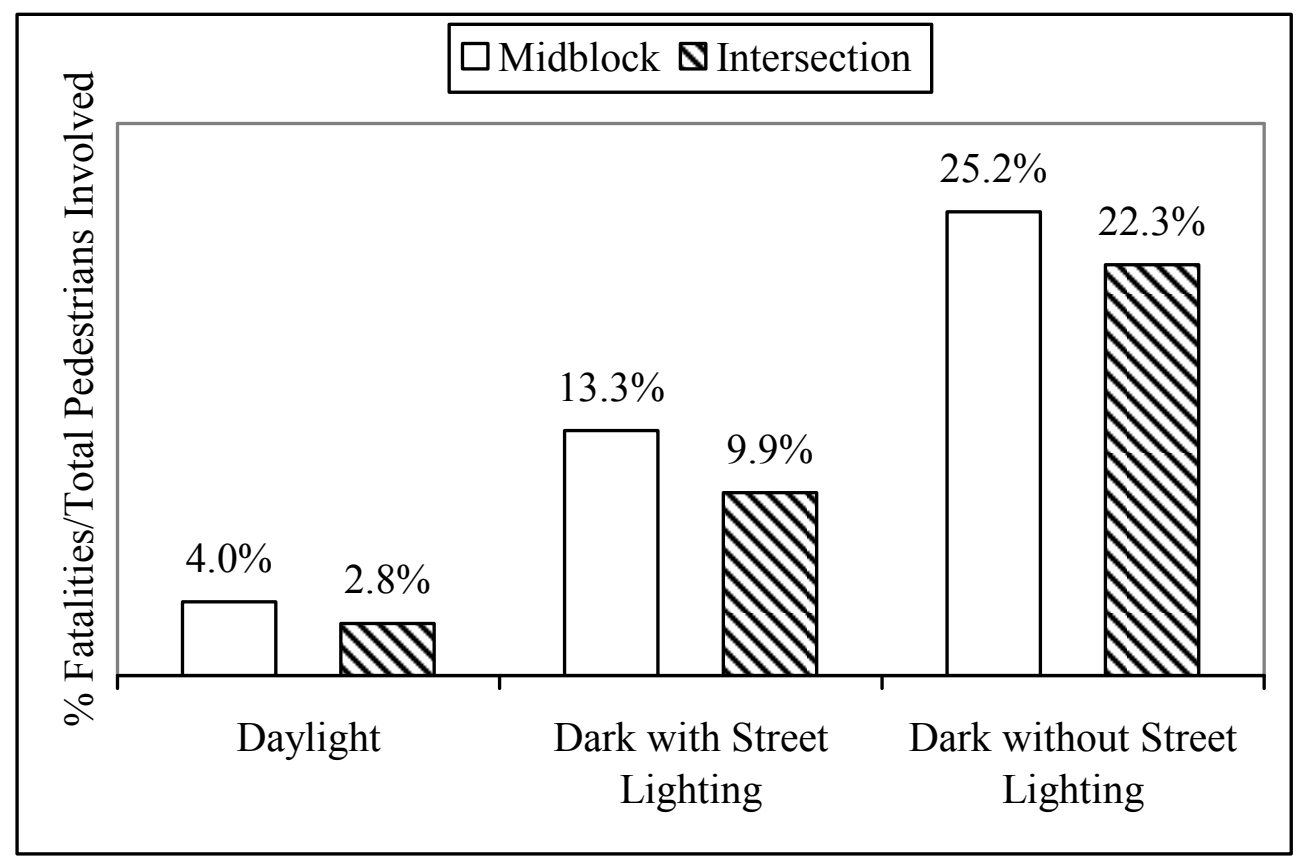

Figure1: Pedestrian Fatality Risk by Crossing Location and Light Condition

Another serious problem with pedestrian safety in Florida relates to light conditions. Again based on data from 1986 through 2003, about 37 percent of all pedestrian crashes occurred while the pedestrians were attempting to cross streets under dark conditions versus daylight conditions. While dark conditions do not represent as large a share of pedestrian crashes as midblock locations, the differential risk across light conditions is significantly higher than that across 
crossing locations. Stated in the probability of a pedestrian getting killed once struck by a vehicle, the fatal injury risk on average is several times higher under dark conditions than under daylight conditions (Figure 1). Earlier studies mention that fatal pedestrian crashes are more likely to occur during nighttime hours while non-fatal pedestrian crashes are more likely to occur during daytime hours (USDOT, 2004). It has been found in previous studies that the probability of a pedestrian getting killed increases at least three times when involved in a nighttime crash compared to a daytime crash (Miles-Doan 1996, Sullivan and Flannagan 2002). A large body of research ascertains the reasons behind the high nighttime fatality risk (Allen, 1970). But previous work has not looked at light conditions and crossing locations in a joint approach.

This paper assesses the role of crossing locations and light conditions in pedestrian injury severity through a multivariate regression analysis to control for many other factors that also may influence pedestrian injury severity. One may not attribute the differential risks in Figure 1 simply to the differences in locations or light conditions. Many other factors that differ across locations or light conditions are likely to have played a role in the observed differential average risks. This paper contributes to the literature in a number of ways. One theoretical advantage of the paper is the use of a reduced-form model of pedestrian injury severity to guide model specification, resulting in unbiased estimates of the effects of crossing locations and light conditions on pedestrian injury severity. One empirical advantage is the use of data for 17 years, resulting in reliable estimates of the effects of crossing locations and light 
conditions on pedestrian injury severity. This is important because of the relatively small number of pedestrian crashes reported each year and the need to estimate the effects of crossing locations and light conditions interactively.

\subsection{Theoretical Framework}

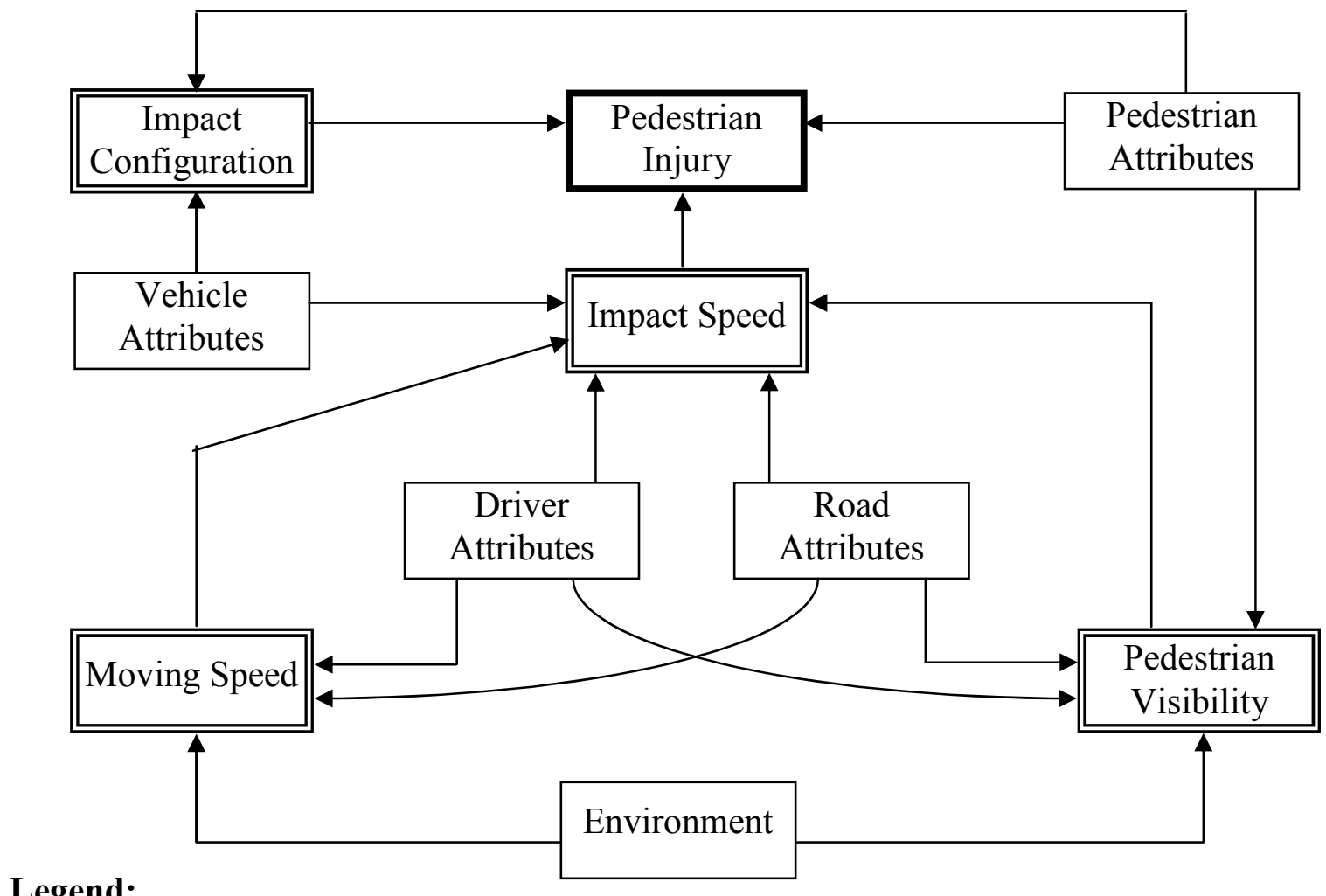

\section{Legend:}

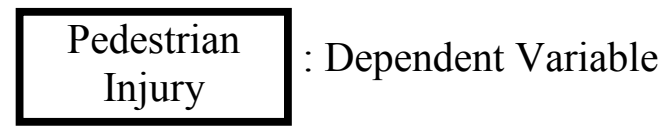

\begin{tabular}{|l|}
\hline Impact Speed : Intermediate Variables \\
\hline
\end{tabular}

\begin{tabular}{l|l}
$\begin{array}{c}\text { Pedestrian } \\
\text { Attributes }\end{array}$ & : Final Independent Variables
\end{tabular}

Figure 2: A Framework on the Determinants of Pedestrian Injury Severity 


\subsubsection{Direct Determinants}

Three sets of factors directly determine the injury severity of a pedestrian once struck by a motor vehicle.

- Impact Speed-The most important of these is the impact speed, which is the speed of the vehicle upon striking the pedestrian (Lee and Abdel-Aty 2005, Sullivan and Flannagan 2002, Jensen 1999, Garder 2004, Pitt et al. 1990). The chance of survival by the pedestrian drops quickly between an impact speed of $20 \mathrm{mph}$ and an impact speed of $40 \mathrm{mph}$ (NHTSA, 1990).

- Impact Configuration-Besides impact speed, one set of determinants relates to impact configuration between the pedestrian and the vehicle (Yang, 2002). This impact configuration includes several aspects, including the angle at which the vehicle strikes the pedestrian (e.g., frontal versus side), the angle at which the pedestrian is struck (i.e., front, back, side), and the height of the impact on the pedestrian.

- Pedestrian Attributes-The final set of determinants relate to the characteristics of the pedestrian. Two pedestrian age groups, the very young (Jensen 1999, LaScala et al. 2001, Al-Ghamdi 2002, Fontaine and Gourlet 1997) and the very old (Lee and Abdel-Aty 2005, Jensen 1999, LaScala et al. 2001, Al-Ghamdi 2002, Fontaine and Gourlet 1997, Zajac and Ivan 2003), are most vulnerable to suffering from severe injuries. Also, male pedestrians, being physically stronger and bigger on average than their female counterparts, may be less likely to sustain severe injuries. 
These three sets of direct determinants are shown in bold in Figure 2. Although, the mass of an involved vehicle is an important determinant of injury severities to both its own occupants and the occupants of the other vehicles involved, it is unlikely to be a significant factor in determining the injury severity of a pedestrian.

\subsubsection{Indirect Determinants}

Policy analysis of pedestrian safety, however, often requires an understanding of indirect determinants of pedestrian injury severity that go beyond the direct determinants. This paper, for example, focuses on the role of crossing locations and light conditions on pedestrian injury severity. But crossing locations and light conditions do not directly affect pedestrian injury severity. These indirect determinants play a role in pedestrian injury severity through their effects on the direct determinants (Figure 2):

- Vehicle attributes may affect both impact configuration and impact speed. High profile vehicles, such as SUVs, are more likely to increase the height of the impact on a pedestrian. Holding other factors constant, on the other hand, heavy vehicles are harder to stop, resulting in a higher impact speed.

- In addition to vehicle attributes, several sets of other factors affect the impact speed of a vehicle. These include the moving speed of the vehicle, driver attributes, road attributes, and pedestrian visibility to the driver. 
- Furthermore, both driver attributes and road attributes affect the moving speed and pedestrian visibility to the driver.

- Pedestrian attributes, such as whether they wear reflective clothing at night, affect pedestrian visibility to the driver.

- Finally, the environment in terms of weather and light conditions can affect both the moving speed of the vehicle and pedestrian visibility to the driver.

In this theoretical framework, crossing locations are part of road attributes, while light conditions are part of the environment. Crossing locations affect pedestrian injury severity most likely through their indirect effects on moving speeds, which in turn affect impact speed. Light conditions, on the other hand, affect pedestrian injury severity largely through their indirect effects on pedestrian visibility to drivers. The following discusses the link between light conditions and impact speed in more detail.

The constraints faced by drivers in recognizing pedestrians at night can be understood by discussing the two types of visual systems used by our eyes for observing and recognizing objects. One is the focal vision controlled by the central retina, which helps in recognizing objects, and another is the ambient vision controlled by peripheral retina, which helps in guiding movements. It has been researched that although focal vision degrades rapidly at night, the ambient system is relatively independent of any errors (Tyrell et al. 2004, Leibowitz and Owens 1986, Leibowitz and Owens 1977, Jeffery and Owens 2001). Due to proper functioning of the guidance mode and rare appearance of pedestrians at 
night, drivers do not realize that their ability to react to an obstruction is adversely affected due to degradation of the recognition mode at night. Apart from that, the visible distance available to the driver at night is fixed due to the use of fixed headlights in the vehicles, which do not respond to changes in vehicle speeds or in the roadway environment. Also, drivers tend to use low beams at night, which reduces available sight distance further. Consequently, at night, often the distance available to drivers for successfully avoiding a crash when a pedestrian appears on road, is shorter than the total stopping sight distance required (leibowitz et al. 1998, Tyrell et al. 2003).

Visual degradation not only affects drivers but also pedestrians. The pedestrian's ability to find a proper gap for crossing roads at night is affected by the indistinctness of a vehicle's position, and the pedestrian's inability to judge the vehicle's actual approaching speed. Also, pedestrians do not realize the visual challenge experienced by drivers at night resulting in them overestimating drivers' observation abilities (Tyrell et al. 2004, Tyrell et al. 2003, Shinar 1984). Allen et al in their study found that the distances at which pedestrians thought they are visible to drivers are far greater than the distance at which they are actually visible to the drivers (Allen, 1970).

\subsection{Methodology}

\subsubsection{Data}

The paper uses an electronic database of all 160,119 pedestrian crashes in the state of Florida reported on its Long Form Police Accident Reports (PARs) in the 
period from 1986 through 2003. It does not include pedestrian crashes reported on Florida's Short Form PARs. The only relevant change over this period is in what is considered a fatal injury. Before 1999, a fatality is a person who died within 90 days of the crash. Since then, a 30-day criterion has been used.

The database uses the KABCO scale for injury severity: possible injury, non-incapacitating injury, incapacitating injury, and fatal injury. The database also includes pedestrian crashes where the pedestrians involved were not injured. In addition to information on injury severity, the database includes a large number of characteristics about the crash, the vehicle, the driver, and the pedestrian. In particular, light conditions are described in five different categories: daylight, dusk, dawn, dark with street lighting, and dark without street lighting. Furthermore, a variable describing pedestrian action at the time of a crash provides information on whether the pedestrian was crossing a road, and where the pedestrian was crossing the road in terms of midblock locations versus intersections.

A total of 78,283 pedestrian crashes are excluded because they do not serve the purpose of the paper. Among these, 77,297 are removed because they relate to pedestrian actions other than street crossing. The other 986 pedestrian crashes are removed because they occurred on freeways. In addition, a total of 23,634 pedestrian crashes are removed because of data problems. Among these, all 8,968 pedestrian crashes for 1990 are removed because data in that year have far more vehicles than drivers involved, indicating a general data problem. Another 9,419 crashes with inconsistent location 
information are also excluded. Besides the variable describing pedestrian action, the database has another variable that describes crash locations. The exclusion criterion in this case of location inconsistency is that these two variables show different locations for a given crash. Furthermore, all 2,817 crashes which occurred under dusk or dawn conditions are removed because the number of pedestrian crashes under these conditions is relatively small for reliable statistical analysis. Finally, 2,430 crashes are excluded due to other data problems, including crashes with unknown light conditions, injury severity, or location. These exclusions result in a total of 58,202 pedestrian crashes for analysis.

\subsubsection{Econometric Model}

This paper uses the ordered probit model. The dependant variable, injury severity, is an ordinal scale, where the relative difference between different injury severities is not well defined. For example, the distance between a possible injury and non-incapacitating injury is different from that between an incapacitating injury and a fatal injury. Previous researcher have used the ordered probit model to analyze crash severity and injury severity of vehicle occupants (McCarthy 2002, Klop and Khattak 1999, O'Donnell and Connor 1996, Duncan et al. 1998, Yamamoto and Shankar 2004, Kockelman and Kweon 2002). More recently, researchers have also used the ordered probit model to model pedestrian injury severity (Lee and Abdel-Aty 2005, Zjac and Ivan 2003). The ordered probit model is built around a latent regression as follows (Greene, 1990): 


$$
y_{i}^{*}=x_{i} \beta+\varepsilon_{i}
$$

where,

- $y_{\mathrm{i}}^{*}$ is the unobserved injury severity for observation $\mathrm{i}$;

- $x_{\mathrm{i}}$ is a row vector of independent variables with 1 in the first column to denote the constant for observation i;

- $\quad \beta$ is a column vector of coefficients with the first row being the constant intercept; and

- $\varepsilon_{\mathrm{i}}$ is the error term that is normally distributed across observations with mean 0 and variance 1.

What the researcher observes is the pedestrian injury severity scale $y$ as follows:

$$
y=\left\{\begin{array}{llll}
0, & \text { if } & \mathrm{y}^{*} \leq 0 & \text { (No Injury) } \\
1, & \text { if } & 0<y^{*} \leq \mu_{1} & \text { (Possible Injury) } \\
2, & \text { if } & \mu_{1}<y^{*} \leq \mu_{2} & \text { (Non-incapacitating Injury) } \\
3, & \text { if } & \mu_{2}<y^{*} \leq \mu_{3} & \text { (Incapacitating Injury) } \\
4, & \text { if } & \mu_{3}<y^{*} & \text { (Fatal Injury) }
\end{array}\right.
$$

where $\mu_{\mathrm{i}}$ 's are unknown thresholds to be estimated with $\beta$. Let $\Phi(\cdot)$ be the cumulative standard normal distribution and $X$ be the matrix of independent variables with 1 in the first column, the probability of a pedestrian suffering each of the injury severities is given by the following:

$$
\begin{aligned}
& \operatorname{Pr}(y=0)=\Phi(-X \beta), \\
& \operatorname{Pr}(y=1)=\Phi\left(\mu_{1}-X \beta\right)-\Phi(-X \beta),
\end{aligned}
$$




$$
\begin{aligned}
& \operatorname{Pr}(y=2)=\Phi\left(\mu_{2}-X \beta\right)-\Phi\left(\mu_{1}-X \beta\right), \\
& \operatorname{Pr}(y=3)=\Phi\left(\mu_{3}-X \beta\right)-\Phi\left(\mu_{2}-X \beta\right), \\
& \operatorname{Pr}(y=4)=1-\Phi\left(\mu_{3}-X \beta\right) .
\end{aligned}
$$

Unlike the commonly used linear regression model, the ordered probit model is non-linear, and its coefficients do not reflect the marginal effect on the dependant variable from one-unit change in any one independent variable. To help interpret the results of the ordered probit model, one common practice is to estimate the marginal effects of the independent variables, and to interpret the ordered probit model through these marginal effects. For a dummy variable, the marginal effect of an independent variable shows the difference in the probability with that variable taking the value of 1 versus 0 . For a continuous independent variable $X_{k}$ and fatal injuries, for example, the marginal effect at the mean of the sample $\bar{X}$ is computed as follows:

$$
\frac{\partial \operatorname{Pr}(y=4 \mid \bar{X})}{\partial X_{k}}=\beta_{\mathrm{k}}\left[\phi\left(\mu_{3}-\bar{X} \beta\right)\right]
$$

where $\phi(\cdot)$ is the standard normal density function.

\subsubsection{Model Specification}

The section describes what variables are included in the row vector of independent variables in the model stated above, and how they are included. The objective is to have a specification that would allow one to estimate the 
differential effects between crossing locations and between light conditions on the probability of pedestrians suffering specific injury severity levels once involved in a motor vehicle crash. The theoretical framework described earlier is used to guide the selection of control variables as well as the approach in which crossing locations and light conditions enter the model.

\subsubsection{Guidance}

The theoretical framework has important implications for model specification. If the objective were to determine the role of impact configuration, or any of the pedestrian characteristics, or impact speed on pedestrian injury severity, one would only need to consider the direct determinants in a model of pedestrian injury severity. However, both crossing locations and light conditions are indirect determinants. A focus on these two indirect determinants requires that the model of pedestrian injury severity exclude impact speed and other intermediate variables (vehicle configuration, moving speed, and pedestrian visibility) and include only final independent variables. In addition to pedestrian attributes, these final independent variables include driver attributes, vehicle attributes, road attributes (including crossing locations), and the environment (including light conditions). While impact speed needs to be excluded, posted speed limit as a part of road attributes needs to be included because it is an important determinant of moving speeds. In mathematical terms, the framework in Figure 2 represents a structural model of pedestrian injury severity, while the focus on the 
effects of crossing locations and light conditions requires the estimation of the reduced-form of the structural model.

\subsubsection{Location and Light Conditions}

In order to measure the effects of both crossing locations and light conditions on the probability of any injury severity, the paper includes five interactive variables between the two locations (midblock and intersection) and the three light conditions (daylight, dark with street lighting, and dark without street lighting):

1. Intersection * Dark with Street Lighting,

2. Intersection * Dark without Street Lighting,

3. Midblock * Daylight,

4. Midblock * Dark with Street Lighting, and

5. Midblock * Dark without Street Lighting.

This specification takes the interaction between intersections and daylight conditions as the base of comparison for all included interactions. Some descriptive statistics of these five interactive variables are shown at the bottom of Table 1 along with the expected sign of their coefficients. 
Table 1: Descriptive Statistics and Expected Direction of Effects

\begin{tabular}{|c|c|c|c|c|}
\hline Variables & Description & Mean & S.D. & Effect \\
\hline AGEGP1_P & 1 if pedestrian is $\leq 10$ years; 0 otherwise & 0.2007 & 0.4005 & + \\
\hline AGEGP2_P & 1 if 11 years $\leq$ pedestrian age $\leq 24$ years; 0 otherwise & 0.2397 & 0.4269 & \pm \\
\hline AGEGP5_P & 1 pedestrian age $\geq 65$ years; 0 otherwise & 0.1477 & 0.3548 & + \\
\hline MALE_P & 1 if pedestrian is male; 0 otherwise & 0.6058 & 0.4887 & - \\
\hline BLACK_P & 1 if pedestrian is Black; 0 otherwise & 0.2949 & 0.4560 & \pm \\
\hline HISPNC_P & 1 if pedestrian is Hispanic; 0 otherwise & 0.0881 & 0.2834 & \pm \\
\hline DISABIL_P & 1 if pedestrian has any physical disability; 0 otherwise & 0.0422 & 0.2011 & + \\
\hline UI_P & 1 if pedestrian was under influence; 0 otherwise & 0.1629 & 0.3692 & + \\
\hline AGEGP1_D & 1 if driver age $\leq 24 ; 0$ otherwise & 0.2502 & 0.4331 & + \\
\hline AGEGP4_D & 1 if driver $\geq 65$ years; 0 otherwise & 0.1118 & 0.3151 & + \\
\hline MALE_D & 1 if driver is male; 0 otherwise & 0.6227 & 0.4847 & + \\
\hline BLACK_D & 1 if driver is Black; 0 otherwise & 0.2411 & 0.4278 & \pm \\
\hline HISPNC_D & 1 if driver is Hispanic; 0 otherwise & 0.0757 & 0.2645 & \pm \\
\hline DISABIL_D & 1 if driver had any physical disability; 0 otherwise & 0.0149 & 0.1210 & + \\
\hline UI_D & 1 if driver was under influence; 0 otherwise & 0.0319 & 0.1758 & + \\
\hline LANES & Number of lanes & 3.4778 & 1.5657 & + \\
\hline UNDIV & 1 if undivided road; 0 otherwise & 0.6173 & 0.4861 & + \\
\hline US & 1 if US owned; 0 otherwise & 0.0659 & 0.2482 & + \\
\hline STATE & 1 if state owned; 0 otherwise & 0.3223 & 0.4674 & + \\
\hline COUNTY & 1 if county owned; 0 otherwise & 0.1908 & 0.3929 & + \\
\hline RURAL & 1 if road in area with population $\leq 2,500 ; 0$ otherwise & 0.3443 & 0.4752 & + \\
\hline POST_SP & Posted speed limit in $\mathrm{mph}$ & 35.2435 & 8.1990 & + \\
\hline RAINY & 1 if it was raining; 0 otherwise & 0.0577 & 0.2332 & \pm \\
\hline FOGGY & 1 if it was foggy; 0 otherwise & 0.0024 & 0.0491 & \pm \\
\hline BIG_VEH & 1 if vehicle is truck, bus, or all terrain; 0 otherwise & 0.1450 & 0.3521 & + \\
\hline YR92TO98 & $\begin{array}{l}1 \text { if crash occurred between } 1992 \text { through 1998; } 0 \\
\text { otherwise }\end{array}$ & 0.4028 & 0.4905 & \pm \\
\hline YR99TO03 & $\begin{array}{l}1 \text { if crash occurred between } 1999 \text { through 2003; } 0 \\
\text { otherwise }\end{array}$ & 0.2594 & 0.4383 & \pm \\
\hline MBDAY & 1 if midblock and daylight; 0 otherwise & 0.4452 & 0.4970 & + \\
\hline MBDRKSL & 1 if midblock and dark with street lights; 0 otherwise & 0.1736 & 0.3788 & + \\
\hline MBDRKNSL & 1 if midblock and dark without street lights; 0 otherwise & 0.0913 & 0.2881 & + \\
\hline ISDRKSL & 1 if intersection an dark with street lights; 0 otherwise & 0.0656 & 0.2475 & + \\
\hline ISDRKNSL & 1 if intersection and dark without street lights; 0 otherwise & 0.0166 & 0.1277 & + \\
\hline
\end{tabular}

Notes: These statistics are for the population of 40,512 pedestrian crashes used in final model estimation, which is 17,690 crashes fewer than what were available after data exclusions described earlier. These crashes are not used in final model estimation due to missing data in at least one of the variables.

Once the overall model is estimated and the marginal effects of individual variables are determined, one can determine the effects of crossing locations and light conditions on the probability of an injury severity. Assuming that $\alpha_{i}$ 
represents the marginal effect of a fatal injury with respect to the i-th interactive variable mentioned earlier, one can determine the effects of crossing locations and light conditions on the probability of a fatal injury. Holding other factors constant, for example, the probability of a fatal injury is expected to be lower at intersections by $-\alpha_{3}$ for daylight conditions, by $\alpha_{1}-\alpha_{4}$ for dark with street lighting conditions, and by $\alpha_{2}-\alpha_{5}$ for dark without street lighting conditions. Similarly, changes in the probability of a fatal injury at either midblock locations or intersections can also be determined between different light conditions. At midblock locations, daylight reduces the probability of a fatal injury by $\alpha_{3}-\alpha_{5}$, and street lighting reduces the probability of a fatal injury by $\alpha_{4}-\alpha_{5}$. At intersections, daylight reduces the probability by $-\alpha_{2}$, and street lighting reduces the probability by $\alpha_{1}-\alpha_{2}$.

Once determined, these differences in the probability of a fatal injury can then be used along with the data from Figure 1 to show the percentage differences in the odds of a pedestrian fatal injury between midblock and intersection locations as well as between light conditions. The odds of something happening is the ratio of the probability of that happening over the probability of that not happening. Figure 1, for example, shows the average probability of a fatal injury under daylight conditions is 4.0 percent at midblock locations and 2.8 percent at intersections. That is, the odds of a fatal injury under daylight conditions is $4.0 /(100-4.0)=0.042$ at midblock locations and $2.8 /(100-2.8)=0.029$ at intersections. Therefore, the odds of a fatal injury is $100(0.029-0.042) / 0.042=$ 31 percent lower at intersections than at midblock locations. For intersections, 
the probability of a fatal injury for each light condition is assumed to be those shown in Figure 1. For midblock locations, the probability for each light condition is that for intersections plus the differences shown in above paragraph. Percent differences in odds are just another useful way to look at the effects of crossing locations and light conditions on pedestrian injury severity.

\subsubsection{Control Variables}

Besides crossing locations and light conditions, all other final independent variables in the framework shown in Figure 2 are considered control variables. They would include all vehicle attributes, all driver attributes, and all pedestrian attributes. With the exception of crossing locations and light conditions, they also include all other road attributes and all weather attributes. The top portion of Table 1 shows some descriptive statistics of all control variables that are available from the original database which are included in the final model.

Pedestrian and Driver Attributes: Two pedestrian age groups, very young (Jensen 1999, LaScala et al. 2001, Al-Ghamdi 2002, Fontaine and Gourlet 1997) and very old (Lee and Abdel_Aty 2005, Zajac and Ivan 2003, Jensen 1999, LaScala et al 2001, Al-Ghamdi 2002, Fontaine and Gourlet 1997) are considered because they are most vulnerable to high severity crashes. Young and male drivers, being typically more aggressive, are likely to be involved in more severe pedestrian crashes. Male pedestrians, being physically stronger than their female counterparts are less likely to sustain severe injury. Pedestrians having any 
physical disability may be more likely to be injured when struck by a vehicle. Drivers having any physical disability may take longer to react, resulting in a higher impact speed. Ethnicity of the pedestrian and the driver has also been included in the model as control variables to avoid any latent bias arising from their omission. One cannot help but notice in Table 1 that the unusually high involvement of blacks both as pedestrians and as drivers relative to the share of blacks in the general population. In fact, blacks represent over 29 percent of the involved pedestrians and over 24 percent of the involved drivers.

Consumption of alcohol by drivers and/or pedestrians is considered an important contributor to higher severity pedestrian crashes. It has been found in previous studies that alcohol consumption by pedestrians considerably increases the probability of getting injured severely or being killed once involved in the crash (Lee and Abdel-Aty 2005, Miles_Doan 1996, Zajac and Ivan 2003, LaScala et al 2001, Fontaine and Gourlet 1997, jehle and Cottington 1988, Öström and Eriksson 2001). It is said that "pedestrians who drink have the judgment skills of a child and the mobility skills of a senior" (FDOT 1996) and are not only more likely to get involved in a crash, but also to sustain more severe injuries once involved. Based on the same reasoning, a drunk driver's ability to react to an obstacle (pedestrian) in the available time is affected adversely and contributes towards higher severity crashes (Zajac and Ivan 2003).

Road Attributes: Vehicular speeds are usually higher on wider roads. Rural roads are associated with higher vehicle speeds and emergency medical 
services are less accessible as they are in rural areas. It has also been found in earlier studies that rural roads are typically associated with more severe pedestrian crashes (Lee and Abdel_Aty 2005, Miles-Doan 1996). Functional classification of roads is unavailable in the data; instead the roadway system has been classified on the basis of ownership and has been used as a substitute. As pointed out already, posted speed limit is an important determinant of average vehicle speeds.

Environment: Rainy and foggy conditions are included for weather conditions. Adverse weather conditions may force drivers to slow down, which is a positive effect on moving speeds. Adverse weather conditions may also make it harder for them to stop, which is a negative effect on impact speed, or for them to see pedestrians, which is a negative effect on their visibility.

Vehicle Attributes: The type of vehicles involved in the crash is also an important determinant of how severely a pedestrian is injured in a crash. Examples are the stiffness and shape of the vehicle front, such as the bumper height, hood height and length, and windshield frame (Yang 2002). Trucks of all size, all terrain vehicles, and buses have been grouped into a category of "big" vehicles and the rest into smaller vehicles. These "big" vehicles are also harder to stop, resulting in a higher impact speed. 
Temporal Attributes: Two dummy variables are included to capture temporal effects on pedestrian injury severity. One covers the period from 1992 to 1998 , and the other from 1999 to 2003 , with the period from 1986 to 1991 as the basis of comparison. These variables are included to capture changes in the transportation system that help reduce pedestrian injury severity but are not controlled for through other control variables. They also are designed to capture any effect of the definitional change of a fatality, made in 1999.

\subsection{Results}

The results are presented in three forms: the estimated model, the derived marginal effects of individual variables for fatal injuries, and the location and lightcondition effects on pedestrian injury severity in terms of both probabilities and odds of fatal injuries.

\subsubsection{Estimated Model}

Table 2 shows the maximum likelihood estimation of the ordered probit model. Repeated from Table 1 are the variable names and their descriptions. For each variable, the table shows the estimated coefficient and its t-statistic. In general, a positive coefficient indicates that an increase in the variable would lead to an increase in pedestrian injury severity. The model is well behaved in general. All variables that have specific expected directions of effects and are statistically significantly have the expected signs. More important, all five interactive variables on crossing locations and light conditions are statistically significant and have the expected direction of effects. Holding other factors constant, pedestrian 
injuries suffered under all other combinations of location and light conditions are

more severe relative to injuries at intersection locations under daylight conditions.

Table 2: Ordered Probit Model of Pedestrian Injury Severity

\begin{tabular}{|c|c|c|c|}
\hline Variables & Description & Coeff. & t-stat \\
\hline ONE & 1 & 1.0032 & 27.64 \\
\hline AGEGP1_P & 1 if pedestrian is under $10 ; 0$ otherwise & 0.0686 & 4.07 \\
\hline AGEGP2_P & 1 if 11 years $\leq$ pedestrian age $\leq 24$ years; 0 otherwise & -0.0705 & -4.94 \\
\hline AGEGP5 P & 1 if pedestrian is over $64 ; 0$ otherwise & 0.3793 & 22.34 \\
\hline MALE_P & 1 if pedestrian is male; 0 otherwise & 0.0146 & 1.31 \\
\hline BLACK_P & 1 if pedestrian is Black; 0 otherwise & -0.1281 & -9.54 \\
\hline HISPNC_P & 1 if pedestrian is Hispanic; 0 otherwise & -0.0152 & -0.72 \\
\hline DISABIL_P & 1 if pedestrian has any physical disability; 0 otherwise & 0.0631 & 2.36 \\
\hline UI_P & 1 if pedestrian was under influence; 0 otherwise & 0.1546 & 9.07 \\
\hline AGEGP1_D & 1 if driver is under $25 ; 0$ otherwise & 0.0890 & 7.09 \\
\hline AGEGP4_D & 1 if driver is over $64 ; 0$ otherwise & 0.0212 & 1.21 \\
\hline MALE_D & 1 if driver is male; 0 otherwise & 0.0211 & 1.88 \\
\hline BLACK_D & 1 if driver is Black; 0 otherwise & -0.0008 & -0.06 \\
\hline HISPNC_D & 1 if driver is Hispanic; 0 otherwise & 0.0526 & 2.35 \\
\hline DISABIL_D & 1 if driver had any physical disability; 0 otherwise & 0.1339 & 3.04 \\
\hline UI_D & 1 if driver was under influence; 0 otherwise & 0.2778 & 8.91 \\
\hline LANES & Number of lanes & 0.0238 & 5.25 \\
\hline UNDIV & 1 if undivided road; 0 otherwise & -0.0056 & -0.43 \\
\hline US & 1 if US owned; 0 otherwise & 0.2520 & 10.17 \\
\hline STATE & 1 if state owned; 0 otherwise & 0.0695 & 4.73 \\
\hline COUNTY & 1 if county owned; 0 otherwise & 0.0902 & 5.38 \\
\hline RURAL & 1 if road in area with population $\leq 2,500 ; 0$ otherwise & 0.0421 & 3.13 \\
\hline POST_SP & Posted speed limit in mph & 0.0204 & 24.77 \\
\hline RAINY & 1 if it was raining; 0 otherwise & -0.0134 & -0.59 \\
\hline FOGGY & 1 if it was foggy; 0 otherwise & 0.2101 & 1.93 \\
\hline BIG_VEH & 1 if vehicle is truck, bus, or all terrain; 0 otherwise & 0.0984 & 6.35 \\
\hline YR92TO98 & 1 if crash occurred between 1992 through 1998; 0 otherwise & -0.0608 & -4.88 \\
\hline YR99TO03 & 1 if crash occurred between 1999 through 2003; 0 otherwise & -0.1111 & -7.85 \\
\hline MBDAY & 1 if midblock and daylight; 0 otherwise & 0.2036 & 14.05 \\
\hline MBDRKSL & 1 if midblock and dark with street lights; 0 otherwise & 0.4623 & 24.93 \\
\hline MBDRKNSL & 1 if midblock and dark without street lights; 0 otherwise & 0.6850 & 28.88 \\
\hline ISDRKSL & 1 if intersection an dark with street lights; 0 otherwise & 0.2929 & 12.08 \\
\hline ISDRKNSL & 1 if intersection and dark without street lights; 0 otherwise & 0.6183 & 14.05 \\
\hline \multicolumn{2}{|l|}{ Observations } & \multicolumn{2}{|c|}{40,512} \\
\hline \multicolumn{2}{|l|}{ Restricted LL } & \multicolumn{2}{|c|}{$-54,292$} \\
\hline \multicolumn{2}{|c|}{ Unrestricted LL } & \multicolumn{2}{|c|}{$-51,451$} \\
\hline
\end{tabular}




\subsubsection{Marginal Effects of Control Variables}

The marginal effects of the control variables on the probability of fatal injuries along with their t-statistics are shown in the top portion of Table 3. The marginal effects of crossing locations and light conditions are discussed in the next subsection. Only the marginal effects for fatal injuries are shown to save space.

Table 3: Marginal Effects on the Probability of a Fatal Injury

\begin{tabular}{|c|c|c|c|}
\hline Variables & Description & $\begin{array}{c}\text { Marginal } \\
\text { Effect }\end{array}$ & t-stat \\
\hline AGEGP1_P & 1 if pedestrian is under $10 ; 0$ otherwise & 0.0088 & 8.03 \\
\hline AGEGP2_P & 1 if 11 years $\leq$ pedestrian age $\leq 24$ years; 0 otherwise & -0.0085 & -6.18 \\
\hline AGEGP5_P & 1 if pedestrian is over $64 ; 0$ otherwise & 0.0575 & 68.69 \\
\hline MALE_P & 1 if pedestrian is male; 0 otherwise & 0.0018 & 1.51 \\
\hline BLACK_P & 1 if pedestrian is Black; 0 otherwise & -0.0153 & -10.06 \\
\hline HISPNC_P & 1 if pedestrian is Hispanic; 0 otherwise & -0.0019 & -1.49 \\
\hline DISABIL_P & 1 if pedestrian has any physical disability; 0 otherwise & 0.0082 & 7.29 \\
\hline UI_P & 1 if pedestrian was under influence; 0 otherwise & 0.0208 & 21.70 \\
\hline AGEGP1_D & 1 if driver is under 25 ; 0 otherwise & 0.0114 & 10.89 \\
\hline AGEGP4_D & 1 if driver is over $64 ; 0$ otherwise & 0.0027 & 2.24 \\
\hline MALE_D & 1 if driver is male; 0 otherwise & 0.0026 & 2.23 \\
\hline BLACK_D & 1 if driver is Black; 0 otherwise & -0.0001 & -0.08 \\
\hline HISPNC_D & 1 if driver is Hispanic; 0 otherwise & 0.0068 & 5.95 \\
\hline DISABIL_D & 1 if driver had any physical disability; 0 otherwise & 0.0183 & 17.94 \\
\hline UI_D & 1 if driver was under influence; 0 otherwise & 0.0418 & 47.47 \\
\hline LANES & Number of lanes & 0.0029 & 5.15 \\
\hline UNDIV & 1 if undivided road; 0 otherwise & -0.0007 & -0.56 \\
\hline US & 1 if US owned; 0 otherwise & 0.0368 & 41.78 \\
\hline STATE & 1 if state owned; 0 otherwise & 0.0088 & 8.16 \\
\hline COUNTY & 1 if county owned; 0 otherwise & 0.0117 & 11.07 \\
\hline RURAL & 1 if road in area with population $\leq 2,500 ; 0$ otherwise & 0.0053 & 4.65 \\
\hline POST_SP & Posted speed limit in $\mathrm{mph}$ & 0.0025 & 19.58 \\
\hline RAINY & 1 if it was raining; 0 otherwise & -0.0017 & -1.32 \\
\hline FOGGY & 1 if it was foggy; 0 otherwise & 0.0305 & 32.51 \\
\hline BIG_VEH & 1 if vehicle is truck, bus, or all terrain; 0 otherwise & 0.0129 & 12.28 \\
\hline YR92TO98 & 1 if crash occurred between 1992 through 1998; 0 otherwise & -0.0075 & -5.43 \\
\hline YR99TO03 & 1 if crash occurred between 1999 through 2003; 0 otherwise & -0.0132 & -9.00 \\
\hline MBDAY & 1 if midblock and daylight; 0 otherwise & 0.0258 & 31.88 \\
\hline MBDRKSL & 1 if midblock and dark with street lights; 0 otherwise & 0.0720 & 79.30 \\
\hline MBDRKNSL & 1 if midblock and dark without street lights; 0 otherwise & 0.1267 & 91.76 \\
\hline ISDRKSL & 1 if intersection an dark with street lights; 0 otherwise & 0.0439 & 50.97 \\
\hline ISDRKNSL & 1 if intersection and dark without street lights; 0 otherwise & 0.1168 & 93.48 \\
\hline
\end{tabular}


Among the control variables, the largest risk factors for fatal injuries facing pedestrians in a decreasing order are: being at least 65 years old, being hit by a driver who is driving under the influence, being involved in a crash on the US road system, walking in foggy conditions, being walking under the influence, being struck by a driver with physical disabilities and being struck by large vehicles. Holding other factors constant, the probability of getting killed once involved in a crash is 5.8 percentage points higher for elderly pedestrians than pedestrians aged from 25 through 64 . While not shown, this is equivalent to an increase of 68 percent in the odds of being killed when struck by a vehicle. Driver being under the influence is a greater fatality risk for pedestrians than pedestrians being under the influence themselves. The probability of a pedestrian getting killed once involved in a crash is 4.5 percentage points higher when being hit by a driver under the influence than when hit by a sober driver. This is equivalent to an increase of 60 percent in the odds of being killed when struck by a vehicle. On the other hand, the probability is 2.1 percentage points higher between a pedestrian who is under the influence and a pedestrian who is sober. This is equivalent to an increase of 40 percent in the odds of being killed. Also, walking in foggy conditions increases the odds of being killed when struck by a vehicle by 42 percent than in non-foggy conditions. On the other hand, crossing in the rain or crossing undivided roads do not appear to be risk factors for pedestrians to be fatally injured once involved in a crash. 


\subsubsection{Locations and Light Conditions}

The marginal effects of the five interactive variables between crossing locations and light conditions on the probability of pedestrian fatal injuries are shown at the bottom of Table 3. To facilitate the discussion, these marginal effects have been translated into the location effects in Table 4 and the light-condition effects in Table 5. The determination of the information in Tables 4 and 5 has been explained in the subsection on model specification earlier.

Table 4: Location Effects: Differences in Probability and Odds of Dying from Crash Involvement between Intersections and Midblock Locations

\begin{tabular}{|l|c|c|}
\hline Light Condition & Probability (Percentage Points) & Odds (Percent) \\
\hline Daylight & $-2.6 \%$ & $-49 \%$ \\
\hline Dark with Lighting & $-2.8 \%$ & $-24 \%$ \\
\hline Dark without Lighting & $-1.0 \%$ & $-5 \%$ \\
\hline
\end{tabular}

The probability of a pedestrian dying from being hit by a vehicle is lower at intersections than at midblock locations for any light condition (Table 4). The difference is 2.6 percentage points under daylight conditions, 2.8 percentage points under dark with street lighting conditions, and 1.0 percentage points under dark without street lighting conditions. While the difference in probability is the largest under dark with street lighting conditions, the odds of sustaining a fatal injury is cut the most under daylight conditions by 49 percent, versus 24 percent under dark with street lighting conditions, and 5 percent under dark without street lighting conditions.

In terms of the probability of a fatal injury, light conditions have a larger effect than locations (Table 5). Daylight reduces the probability of a fatal injury 
by 10.1 percentage points at midblock locations and 11.7 percentage points at intersections, while street lighting reduces the probability of a fatal injury by 5.5 percentage points at midblock locations and 7.3 percentage points at intersections. In terms of the odds of a fatal injury, however, the difference is less clear cut. Daylight does have a larger effect on the odds of a fatal injury than the effect of locations under any light condition. The effect of street lighting is larger than the effect of locations under dark conditions but is comparable with the effect of locations under daylight conditions.

Table 5: Effects of Daylight and Street Lighting: Differences in Probability and Odds of Dying from Crash Involvement

\begin{tabular}{|l|l|c|c|}
\hline Effects of & Location & Probability (percentage points) & Odds (percent) \\
\hline \multirow{2}{*}{ Daylight } & Midblock & $-10.1 \%$ & $-75 \%$ \\
\cline { 2 - 4 } & Intersection & $-11.7 \%$ & $-83 \%$ \\
\hline \multirow{2}{*}{ Street Lighting } & Midblock & $-5.5 \%$ & $-42 \%$ \\
\cline { 2 - 4 } & Intersection & $-7.3 \%$ & $-54 \%$ \\
\hline
\end{tabular}

Notes: The effect of daylight is calculated as the difference in marginal effects between daylight and dark without street lighting. The effect of street lighting is calculated as the difference in marginal effects between dark with street lighting and dark without street lighting.

The effect of street lighting is smaller than the effect of daylight (Table 5). Street lighting reduces the probability of a fatal injury by 5.5 percentage points at midblock locations and 7.3 percentage points at intersections. In comparison, the reductions from daylight are 10.1 percentage points at midblock locations, and 11.7 percentage points at intersections. Similarly, street lighting results in a reduction in the odds of a fatal injury around 50 percent, versus a reduction of around 80 percent from daylight. 
The effect of light conditions is greater at intersections than at midblock locations (Table 5). Daylight reduces the probability of a fatal injury by 11.7 percentage points at intersections versus 10.1 percentage points at midblock locations. In terms of the odds of a fatal injury, the reductions are 83 percent at intersections and 75 percent at midblock locations. Similarly, street lighting reduces the probability of a fatal injury by 7.3 percentage points at intersections but 5.5 percentage points at midblock locations. The effect of street lighting in terms of the odds of a fatal injury is a reduction of 54 percent at intersections but 42 percent at midblock locations.

\subsection{Conclusions}

Applying the ordered probit model to crash data from 1986 to 2003 in Florida, the paper assesses the role of crossing locations and light conditions on the injury severity of pedestrians once being struck by motor vehicles while crossing roads. The empirical model is well behaved. It includes pedestrian attributes, driver attributes, road attributes, vehicle attributes, and weather conditions as control variables. All control variables having a specific expected direction of effects and are statistically significantly have the expected signs. More importantly, all five interactive variables on crossing locations and light conditions are found to be statistically significant and also have the expected direction of effects.

The empirical model provides insights on the role of various control variables on pedestrian injury severity. The largest risk factors for fatal injuries facing pedestrians when struck by a vehicle in a decreasing order are: being at 
least 65 years old, being hit by a driver who is DUI, being involved in a crash on the US road system, walking in foggy conditions, being WUI, being hit by a driver with physical disabilities, and being hit by large vehicles. Holding other factors constant, for example, the odds of getting killed when struck by a vehicle is 68 percent higher for elder pedestrians than for 25-64 old pedestrians. Being under the influence by drivers is a greater fatality risk for pedestrians than being under the influence by pedestrians themselves. The odd of a pedestrian getting killed is 60 percent higher when struck by a driver under the influence than when struck by a sober driver. On the other hand, the odd is 40 percent higher between a pedestrian who is under the influence and a pedestrian who is sober. Also, walking in foggy conditions increases the odds of being killed when struck by a vehicle by 42 percent than in non-foggy conditions. However, crossing in the rain or crossing undivided roads does not appear to be risk factors for pedestrians to be fatally injured once involved in a crash.

More important, the results provide new insights on the role of crossing locations and light conditions on pedestrian injury severity. In terms of crossing locations, the probability of a pedestrian dying when struck by a vehicle is higher at midblock locations than at intersections for any light condition. In fact, the odds of sustaining a fatal injury at intersections is 49 percent lower than at midblock locations under daylight conditions, 24 percent lower under dark with street lighting conditions, and 5 percent lower under dark without street lighting conditions. Relative to dark conditions without street lighting, daylight reduces the odds of a fatal injury by 75 percent at midblock locations and by 83 percent at 
intersections, while street lighting reduces the odds by 42 percent at midblock locations and by 54 percent at intersections.

Like most previous work, this paper also relies on electronic data from accident reports completed by investigating officers at the time of a crash. It is well established that traffic accident reports suffer from inconsistencies and inaccuracies due to judgmental and reporting discrepancies, including the injury severity of pedestrians involved (Agran et al 1990). Lighting conditions from street lights under dark conditions do not reflect the quantity and quality of light. Additional errors may be introduced when information from the accident reports is entered into electronic databases. Furthermore, some pedestrian crashes are either un-reported or reported but not made electronically available.

Like most previous work, this paper focuses on the resulting injury severity of the pedestrian after a crash has already occurred. In order to assess the overall roles of crossing locations and light conditions in pedestrian safety for street crossing, the relative probability of a pedestrian getting involved in crashes at different crossing locations and under different light conditions also needs to be considered. Such a broader consideration would require data on pedestrian exposure to vehicle traffic while crossing streets. One good measure would be pedestrian crossing volumes at the locations where pedestrian crashes occurred and at the times when these pedestrian crashes occurred. However, such exposure data are unavailable, and the probability of a pedestrian getting involved in a crash has not been incorporated in this study. 
This paper has several advantages over previous work, however. One theoretical advantage of the paper is the use of a reduced-form model of pedestrian injury severity to guide model specification, resulting in unbiased estimates of the effects of crossing locations and light conditions on pedestrian injury severity. One empirical advantage is the use of data for 17 years, resulting in reliable estimates of the effects of crossing locations and light conditions on pedestrian injury severity. This is important because of the relatively small number of pedestrian crashes reported each year, the potential errors in traffic accident reports, and the need to estimate the effects of crossing locations and light conditions interactively. 


\section{CHAPTER 3 \\ CONCLUSIONS}

The study established a relationship between crossing locations, light conditions, and the injury severity of pedestrians involved in crashes while attempting to

cross a street. This is a first attempt to compare the injury severity of the pedestrians involved in crashes at midblock locations to the intersection locations. It has been clearly quantified in the present analysis that once involved in a crash, a pedestrian is likely to suffer more severe injury at midblock locations under all light conditions. The role played by daylight and streetlights has also been quantified in the study.

The study does a post analysis of the pedestrian crashes which have already occurred and does not account for the probability of getting involved into a crash while crossing at midblock locations versus intersection locations. A good way of looking at the relative effects will be to include the probability of getting involved in the crash along with analyzing the injury severities after the crash occurs. However, such exposure data is very difficult to obtain.

As a follow up to this study, the role of midblock crosswalk can be established using the same dataset. The data set, starting 1991 classifies the midblock crash locations into crosswalk and non-crosswalk locations which can 
be helpful in studying such effects. With policy point of view, this follow up study in conjunction with the role of street lights established in the present study can be used to recommend the installation of street lights and placement of midblock crosswalk. 


\section{REFERENCES}

Agran P.F., Castillo D.N., Winn D.G.; 1990. Limitations of Data Compiled from Police Reports on Pediatric Pedestrian and Bicycle Motor Vehicle Events. Accident Analysis and Prevention 22 (4) 361-370.

Al-Ghamdi, A.S., 2002. Pedestrian-vehicle crashes and analytical techniques for stratified contingency table. Accident Analysis and Prevention 34 (2) 205-214.

Allen M.J ; Vision and highway safety; (1970).

Duncan, C.S., Khattak, A.J., Council, F.M., 1998. Applying the ordered probit model to injury severity in truck-passenger car rear-end collisions. Transportation Research Record 1635, 63-71.

FDOT, 1996. Pedestrian Planning and design guidelines.

Fontaine, Hélène and Gourlet, Yves, 1997. Fatal pedestrian accidents in France: A typological analysis. Accident Analysis and Prevention 29 (3) 303-312.

Garder, P E; 2004. The impact of speed and other variables on pedestrian safety in Maine; Accident Analysis and Prevention 36 (4) 522-542.

Greene, Williams, 1990. Econometric Analysis.

Jeffrey A., Owens, D.A., 2001. The twilight envelope: A user centered approach to describing roadway illumination at night; Human Factors, 43 (4) 620-630.

Jehle D, Cottinton E, 1988. Effect of alcohol consumption on outcome of pedestrian victims. Annals of emergency medicine, Vol. 17 Issue 9.

Jensen, S.U., 1999. Pedestrian safety in Denmark. Transportation Research Record, 1674, 61-69.

Klop, J.R., Khattak, A.J., 1999. Factors influencing bicycle crash severity on twolane, undivided roadways in North Carolina. Transportation Research Record 1674, 78-85. 
Kockelman, K.M., Kweon, Y.J., 2002. Driver Injury Severity: an application of ordered probit models. Accident Analysis and Prevention 27 (3) 313-321.

LaScala E. A., Fred W. Johnson, and Paul J. Gruenewald, 2001. Neighborhood Characteristics of Alcohol-Related Pedestrian Injury Collisions: A Geostatistical Analysis. Prevention Science, Vol 2, No. 2.

Lee Chris, Abdel-Aty Mohamed, 2005. Comprehensive analysis of vehiclepedestrian crashes at intersections in Florida. Accident Analysis and Prevention 37 (4) $775-786$.

Leibowitz H.W and Owens D.A, 1977. Nighttime Driving Accidents and Selective Visual Degradation; Science, New Series, Vol. 197, No. 4302, 422-423.

Leibowitz H.W., Owens, D.A, 1986. We Drive by night; and when we do we often misjudge our visual abilities, courting disaster; Psychology Today Jan 1986 v20 p54 (5).

Leibowitz, H.W., Owens D.A., Tyrrell R.A., 1998. The assured clear distance ahead rule: Implications for nighttime traffic safety and the law. Accident Analysis and Prevention 30 (1) 93-99.

McCarthy Patrick, 2002. Driving Under the Influence and Older Driver-Involved Crash Severity: An Ordered Probit Analysis.

http://www.econ.gatech.edu/papers/mccarthy_ord_prbt_nih2000_SOEWebst_09 1402.pdf

Miles-Doan, Rebecca, 1996. Alcohol use among pedestrians and the odds of surviving an injury: Evidence from Florida law Enforcement Data; Accident Analysis and Prevention 28 (1) 23-31.

National Highway Traffic Safety Administration (NHTSA), 1999. Literature Review on Vehicle Travel Speeds and Pedestrian Injuries, U.S. Department of Transportation, Washington, D.C.

National Highways and Traffic Safety Administration NHTSA: Traffic Safety Facts 2003.

O'Donnell, C.J., Connor, D.H., 1996. Predicting the severity of motor vehicle accident injuries using models of ordered multiple choice Accident Analysis and Prevention 28 (6), 739-753.

Öström, Mats, and Eriksson, Anders, 2001. Pedestrian fatalities and alcohol. Accident Analysis and Prevention 33 (2) 173-180.

Pitt, R., Guyer, B., Hsieh, C., Malek, M., 1990. The severity of pedestrian injuries in children: an analysis of the pedestrian injury causation study. Accident Analysis and Prevention 22 (6), 549-559. 
Shinar, D., 1984. Actual versus estimated night-time pedestrian visibility. Ergonomics, 27, 863-871.

Sullivan, J.M., Flannagan, M.J., 2002. The role of ambient light level in fatal crashes: inferences from daylight saving time transition Accident Analysis and Prevention 34 (4) 487-498.

Tyrrell, R. A., Wood, J. M., and Carberry T. P., 2003. On-road measures of pedestrians' estimates of their own nighttime visibility: effects of clothing, beam, and age. Presented at the $81^{\text {st }}$ Transportation Research Board Annual Meeting.

Tyrrell, R.A., Brooks, J.O, Joanne M.W., Carberry, T.P., 2004. Nighttime conspicuity from the pedestrian's perspective. Presented at the $83^{\text {rd }}$ Transportation Research Board Annual Meeting.

US DOT, FHWA; A Review of Pedestrian Safety Research in the United States and Abroad; January 2004.

Yamamoto T., Shankar V.N, 2004. Bivariate ordered-response probit model of driver's and passenger injury severities in collision with fixed objects. Accident Analysis and Prevention 36 (5), 869-876.

Yang, Jikuang, 2002. Review of Injury Biomechanics in Car-Pedestrian Collisions. Crash Safety Divisions, Chalmers University of Technology, Sweden.

Zajac S.S, Ivan J.N., 2003. Factors influencing injury severity of motor vehiclecrossing pedestrian crashes in rural Connecticut. Accident Analysis and Prevention 35 (3) 369-379. 
APPENDICES 


\section{Appendix A: Letter of Acceptance for Publication}

Date Sent: 3/14/2006 10:57:39 AM

From: Richard F. Pain (jcole@nas.edu)

To: Naved Alam Siddiqui (siddiqui@cutr.usf.edu)

Subject: TRB Paper 06-1601 Final Publication Acceptance

\section{Dear Author:}

On behalf of the Transportation Research Board (TRB), I am pleased to inform you that the paper listed below has been accepted for publication in the Transportation Research Record (TRR), Journal of the Transportation Research Board. Please submit your final revised paper to TRB by April 1, 2006 using the following website and login information:

http://www.trb.org/submissions/default.asp?event=68 and the login name and password associated with your siddiqui@cutr.usf.edu email address. If you have forgotten your login name and password or the one you have been using no longer works, you may reset it by clicking on the "I Forgot My Login Information" link on the login page, entering siddiqui@cutr.usf.edu, and following the instructions. All papers accepted for publication must be submitted again to TRB, even if no changes were requested after the peer review. If it is necessary to mail your manuscript, please send the electronic file (MS Word 6.0 or newer) on a 3.5" diskette, Zip disk, or CD with a paper copy of the file content to the TRB Publications Office at the address shown below. Please make sure you do not 


\section{Appendix A: (Continued)}

inadvertently resubmit the version of your paper that appeared on the Annual Meeting Compendium of Papers CD-ROM.

Please carefully review the "Information for Authors" (available at http://www.trb.org/Guidelines/Authors.pdf) to ensure that your manuscript submittal is complete and prepared in accordance with the publication specifications. To simplify formatting of the manuscript, use 1-inch margins at the top and left of each page. To prevent problems in reproduction of the figures and tables, the use of color and screens should be avoided. IMPORTANT: Place each figure or table on a page by itself at the end of the manuscript file (figures and tables should not be embedded in the text) or, if necessary, in a file separate from the text file. Include a list of all figure captions and table titles.

If your manuscript exceeds the length limit shown below, it will be returned to you for revision, which will delay publication. The paper's word count should be indicated on the first page of the manuscript. TRB has a rigid production schedule to ensure that papers are published within a reasonable time period. To meet this schedule, the due date shown below must be observed. If your manuscript is received after this date, publication may be deferred until the next publication year or cancelled. Earlier submission is encouraged. Please call me if you have any questions regarding the publication of your paper.

Sincerely,

Richard F. Pain 


\section{Appendix A: (Continued)}

Transportation Research Board

Technical Activities (Div A)

500 Fifth Street NW

Washington, DC 20001

202-334-2964

202-334-2003

jcole@nas.edu

Length Limit: 7500 (tables and figures count for 250 words each)

Due Date: April 1, 2006

Reviewing Committee: ANF10 - Pedestrians

TRB Paper Number: 06-1601

Paper Title: Crossing Locations, Light Conditions, and Pedestrian Injury Severity

Publications Address:

Transportation Research Board

Publications Office

Keck Center, Room 476

500 5th Street, NW

Washington, DC 20001 